\title{
Calcofluor White Staining Method
}

National Cancer Institute

\section{Source}

National Cancer Institute. Calcofluor White Staining Method. NCI Thesaurus. Code C85577.

A microscopy staining method that utilizes calcofluor, a fluorescent dye that preferentially stains chitin and cellulose on the cell walls of fungi, parasites, and some cysts of the Pneumocystis genus. 